\title{
ESTILOS DE VIDA DE LOS USUARIOS DE LAS SALAS DE CONSUMO HIGIÉNICO DE BARCELONA
}

\section{LIFESTYLES OF DRUG CONSUMPTION ROOMS`USERS IN BARCELONA}

\author{
Rafael Clua García \\ Facultat de Ciències de la Salut de Manresa, Universitat de Vic $\neg-$ Universitat Central de Catalunya, España
}

\section{Abstract}

Studies focusing on the lifestyle of the Barcelona drug consumption rooms' users are inexistent. However, acknowledging these lifestyles is essential to understand the social and cultural factors shaping their behaviours, thus improving our knowledge about the use of harm reduction programs and treatment of drug addiction. To examine these issues, a qualitative study was conducted by combining participant observation in five drugs consumption rooms with semi-directed interviews with 16 drugs users. By comparing users' characteristics as wells analysing the different consumption patterns and the prevention measures used, we identify four different lifestyles: "rutero", "parroquiano", "pasajero" and "fiestero". These findings expand our understanding of the social and sanitary needs as well as our knowledge of drugs consumers' motivations. These aspects are crucial to design policies and programs aiming to harm reduction and to improve existing ones.

Keywords: Cocaine, heroin dependence, life style, qualitative research, supervised injecting facilities

\section{Resumen}

En la actualidad, no se conocen investigaciones sobre los estilos de vida de los usuarios de las salas de consumo higiénico $(\mathrm{SCH})$ de Barcelona. Dicho aspecto es fundamental para profundizar en los factores socioculturales que determinan los comportamientos de los consumidores de drogas que acuden a estos dispositivos y aumentar el conocimiento del uso que hacen de los programas de reducción de daños y los tratamientos para las drogodependencias. Con el fin de investigar esta cuestión, se realizó un estudio cualitativo combinando la observación participante en cinco SCH y entrevistas semiestructuradas a 16 usuarios de drogas. Al comparar las características de los usuarios y analizar las formas de consumo y las medidas de prevención que utilizan, se identificaron cuatro estilos de vida: "rutero", "parroquiano", "pasajero" y "fiestero". Estos hallazgos amplían nuestra comprensión de las necesidades sociales y sanitarias, así como nuestro conocimiento de las motivaciones de los consumidores de drogas. Estos aspectos son cruciales para el diseño de políticas y programas de reducción de daños más eficaces.

Palabras clave: Cocaína, dependencia de heroína, estilo de vida, investigación cualitativa, salas de consumo higiénico 
En 2001 el ayuntamiento de Barcelona apostó por la instalación de salas de consumo higiénico ( $\mathrm{SCH}$ ), dispositivos para consumir heroína y cocaína donde se garantiza material higiénico y la supervisión de profesionales para actuar en caso de sobredosis u otras complicaciones. En 2015, se contaba con 10 SCH en Barcelona y sus alrededores, 8 integradas en centros ambulatorios y 2 en unidades móviles (Clua, 2015). Diversas revisiones bibliográficas (Clua, 2015; Hedrich, Kerr y Dubois-Arber, 2010; Noël et al., 2009; Potier et al., 2014) han demostrado que las SCH son efectivas para evitar las muertes por sobredosis, disminuir la transmisión de enfermedades infectocontagiosas (VIH y hepatitis $\mathrm{B}$ y $\mathrm{C}$ ), promover la asistencia sociosanitaria y mejorar el orden público.

Sin embargo, estudios recientes concluyen que los usuarios de estos dispositivos mantienen conductas de riesgo, no tienen cubiertas sus necesidades básicas y que es necesario mejorar el acceso a los programas de reducción de daños para evitar las consecuencias negativas del consumo de drogas (Clua, 2015; Folch et al., 2012). Se estima que los usuarios de las SCH de Barcelona son hombres en más de un $80 \%$, de edades comprendidas, mayoritariamente, entre los 30 y los 40 años, y más del $40 \%$ son inmigrantes. Manifiestan trayectorias de más de 10 años de consumo de drogas, en su mayoría de heroína y/o cocaína por vía parenteral. En relación con el estado serológico, se registra una prevalencia de más del 30\% por VIH y del $70 \%$ por hepatitis C (alrededor del 30\% coinfectados por el $\mathrm{VIH}$ y el VHC). A nivel social, se trata de una población con bajo nivel de instrucción, una baja vinculación al mundo laboral y sin vivienda fija o que viven en la calle. Dadas dichas condiciones sociales, muchos usuarios para la obtención de dinero utilizan medios ilegales (mendicidad, trapicheos, hurtos y robos) que conlleva a tener procesos judiciales abiertos (Clua, 2015; Folch et al., 2012).

En la actualidad, no existen estudios que hayan empleado una perspectiva holística para interpretar los factores socioculturales que determinan los comportamientos de riesgo y daño de los consumidores de drogas y para conocer las dificultades de acceso a los programas de reducción de daños. Para resolver este problema, en esta investigación se tomó la perspectiva del modelo sociocultural para conocer los estilos de vida de los usuarios de las SCH. Dicho modelo pone atención en la cultura y el contexto social, considerando el concepto "adicción" más allá de una categoría exclusivamente "natural" o "biológica" que se entrecruza con la cultura, la política, los intereses sociales y económicos (Apud y Romaní, 2016; Romaní, 2010; Singer, 2012). En este marco, mediante el concepto "estilo de vida" se procura la interpretación global de las formas de vivir de un grupo social determinado para describir las prácticas y discursos que utilizan para su perdurabilidad (Menéndez, 1998). De este modo, el estilo de vida se toma como una noción construida entre las experiencias y las decisiones de los sujetos y las disposiciones y los procesos de socialización regulados desde variables estructurales (Dumont y Clua, 2015). Siguiendo a Dumont y Clua (2015), las siguientes dimensiones permiten describir los estilos de vida:

a) Temporalidad. Los estilos de vida cambian y se transforman a lo largo del tiempo mediante la apropiación de nuevos elementos y la transformación de las acciones y formas de pensar llevadas a cabo por los individuos y los grupos sociales de un contexto determinado.

b) Visibilidad. Los estilos de vida se configuran a través de elementos materiales y simbólicos que son visibles y reconocibles entre sus miembros. No obstante, en ciertos estilos de vida estigmatizados, estos elementos pueden ser ocultados con diferentes estrategias que impiden el reconocimiento entre los individuos de un colectivo.

c) Espacial. Los diferentes estilos de vida se constituyen con la apropiación y negociación de los espacios simbólicos, sociales y territoriales.

d) Compromiso. Los individuos y grupos sociales contraen, de forma consciente e inconsciente, una serie de conductas para construir su identidad social y las dinámicas de actuación con diferentes intensidades.

e) Reflexividad. Se refiere a la sucesión de vínculos que construyen los individuos y grupos sociales con sus prácticas culturales y el mundo social que les rodea.

Desde esta mirada, se analizan las prácticas y discursos de las personas, considerando los estilos de vida como una decisión racional a la vez que se indaga en las condiciones materiales, sociales y culturales de las que dispone para la adopción de unos comportamientos determinados (Álvarez, 2012). En relación a esta idea, las diferentes prácticas y discursos hacia el consumo de drogas son determinadas subjetivamente por los consumidores a partir de la experiencia del riesgo 
(Rhodes, 2009; Sepúlveda y Romaní, 2013) y deben ser analizadas en los múltiples contextos para dar una respuesta sociosanitaria más efectiva. Dado lo anterior, el objetivo de este artículo es analizar los estilos de vida de los usuarios de las SCH de Barcelona focalizando en la intensidad del consumo de drogas y la adherencia a estos dispositivos, elementos clave para el diseño de políticas y programas de reducción de daños y tratamientos más eficaces.

\section{MÉTODO}

\section{Diseño}

La presente investigación parte de un paradigma cualitativo de carácter etnográfico. La etnografía permite un acercamiento a la realidad, estableciendo una interacción directa con los diferentes actores durante un período, más o menos largo, en el ambiente natural donde se produce el consumo de drogas (Bourgois y Schonberg, 2009; Carlson et al., 2009; Romaní, 2010; Singer, 2012). Esta perspectiva hizo posible profundizar y comparar las características de los estilos de vida de los consumidores de drogas dentro de su cotidianidad. La investigación se realizó entre noviembre de 2012 y octubre de 2014 en 5 centros de atención a las drogodependencias con SCH de Barcelona (Tabla 1) y sus alrededores (vía pública), situadas en tres zonas con una fuerte escena de venta de drogas por las que circulan el mayor número de consumidores de drogas de esta área metropolitana.

Tabla 1. Salas de consumo higiénico estudiadas (ASPCat, 2016; Clua, 2015)

\begin{tabular}{|c|c|c|c|c|c|}
\hline & SAPS & CAS Baluard & $\begin{array}{l}\text { Unidad móvil de } \\
\text { Zona Franca }\end{array}$ & CAS Fòrum & $\begin{array}{c}\text { Centro sociosanitario } \\
\text { "El Local" }\end{array}$ \\
\hline Ubicación & $\begin{array}{c}\text { Centro de la ciudad de } \\
\text { Barcelona }\end{array}$ & $\begin{array}{c}\text { Centro de la ciudad de } \\
\text { Barcelona }\end{array}$ & $\begin{array}{c}\text { Barrio periférico de } \\
\text { Barcelona }\end{array}$ & $\begin{array}{c}\text { Barrio periférico de } \\
\text { Barcelona }\end{array}$ & $\begin{array}{l}\text { Extrarradio, barrio de } \\
\text { Sant Adrià de Besòs }\end{array}$ \\
\hline Organización & Creu Roja & ASPB/ABD & ASPB/ABD & Parc Sanitari Mar & $\begin{array}{l}\text { Ajuntament de Sant } \\
\text { Adrià de Besòs / IPSS }\end{array}$ \\
\hline $\begin{array}{l}\text { Horario de } \\
\text { apertura }\end{array}$ & $\begin{array}{c}\text { De lunes a jueves de } \\
18 \text { a 1h., viernes de } 13 \\
\text { a } 20 \mathrm{~h} .\end{array}$ & $\begin{array}{c}\text { De lunes a viernes de } 7 \text { a } \\
\text { 22h., fines de semana de } 8 \\
\text { a } 19 \mathrm{~h}\end{array}$ & $\begin{array}{l}\text { De lunes a viernes de } \\
\qquad 14 \text { a 21:30h. }\end{array}$ & $\begin{array}{l}\text { De lunes a domingo } \\
\text { de } 12: 15 \text { a } 18: 45 \mathrm{~h} .\end{array}$ & $\begin{array}{c}\text { De lunes a viernes de } 11 \\
\text { a 19h., fines de semana } \\
\text { de } 11 \text { a } 15 \mathrm{~h} .\end{array}$ \\
\hline Plazas de la SCH & $\begin{array}{l}2 \text { para el consumo por } \\
\text { vía parenteral }\end{array}$ & $\begin{array}{c}6 \text { para el consumo por vía } \\
\text { parenteral } \\
5 \text { para el consumo por vía } \\
\text { pulmonar }\end{array}$ & $\begin{array}{l}3 \text { para el consumo por } \\
\text { vía parenteral }\end{array}$ & $\begin{array}{c}1 \text { para el consumo por } \\
\text { vía parenteral }\end{array}$ & $\begin{array}{l}8 \text { para el consumo por } \\
\text { vía parenteral }\end{array}$ \\
\hline $\begin{array}{l}\text { Programas y } \\
\text { áreas del } \\
\text { dispositivo }\end{array}$ & $\begin{array}{c}\text { PIJ, enfermería y "calor } \\
\text { y café" }\end{array}$ & $\begin{array}{l}\text { PIJ, PMM, enfermería y } \\
\text { "calor y café" }\end{array}$ & $\mathrm{PIJ}$ & $\begin{array}{l}\text { PIJ, PMM, enfermería y } \\
\text { "calor y café" }\end{array}$ & $\begin{array}{c}\text { PIJ, enfermería y "calor y } \\
\text { café" }\end{array}$ \\
\hline $\begin{array}{l}\text { Número de } \\
\text { usuarios (2015) }\end{array}$ & 415 & 1.875 & 94 & 625 & 1.967 \\
\hline
\end{tabular}

Nota: ASPB = Agència de salut pública de Barcelona; ABD = Associació Benestar i Desenvolupament; CAS = centro de atención y seguimiento a las drogodependencias; IPSS = Institut per a la promoció social i de la salut; PIJ = programa de intercambio de jeringuillas; PMM = programa de mantenimiento con metadona

\section{Participantes}

La captación de los participantes del estudio se realizó durante la fase de familiarización de los diferentes ambientes de observación. Se llevó a cabo un muestreo cualitativo y teórico (Martínez-Salgado, 2012). Este no persiguió agrupar a los participantes en base a criterios estadísticos, sino a interpretar el fenómeno estudiado incluyendo tanto los "casos comunes" como aquellos "menos habituales" que transitan por estos dispositivos, obteniendo un mayor alcance en el diseño de los tipos sociales de consumidores.

Durante el trabajo de campo se contactó con más de un centenar de consumidores de drogas, de manera directa y por medio de la técnica "bola de nieve" (Shaghaghi, Bhopal y Sheikh, 2011), a través de los usuarios y los profesionales, siendo 16 usuarios los que fueron entrevistados de manera formal. Participaron 9 hombres, 5 mujeres y 2 transexuales con edades comprendidas entre 30 y 50 años, de los cuáles 6 eran consumidores de heroína, 3 de cocaína y 7 de ambas, y 12 eran consumidores por vía parenteral, 1 por vía pulmonar y 3 por ambas (Tabla 2). 
Tabla 2. Características de los usuarios de las SCH entrevistados

\begin{tabular}{|c|c|c|c|c|c|c|c|}
\hline $\begin{array}{l}\text { Usuario y } \\
\text { tipología }\end{array}$ & Sexo & $\begin{array}{l}\text { Año y lugar de } \\
\text { nacimiento }\end{array}$ & Ingresos & Vivienda & $\begin{array}{l}\text { Tipo de } \\
\text { consumo }\end{array}$ & Patologías & $\begin{array}{c}\text { Seguimiento } \\
\text { en centro de salud }\end{array}$ \\
\hline $\begin{array}{l}\text { Ramón } \\
\text { Rutero }\end{array}$ & Varón & 1962, Córdoba & $\begin{array}{l}\text { PNC, hurtos y } \\
\text { trapicheos }\end{array}$ & Piso compartido & $\begin{array}{c}\text { Heroína, VI y } \\
\text { VP }\end{array}$ & $\mathrm{VHC}$ & Irregular en CAS \\
\hline $\begin{array}{l}\text { Juanjo } \\
\text { Parroquiano }\end{array}$ & Varón & 1964, Barcelona & Mendicidad & Calle, pensiones & Heroína, VI & VHC y VIH & Centro de día \\
\hline $\begin{array}{l}\text { Julián } \\
\text { Parroquiano }\end{array}$ & Varón & $\begin{array}{l}\text { 1970, } \\
\text { Sevilla }\end{array}$ & $\begin{array}{l}\text { Hurtos y } \\
\text { trapicheos }\end{array}$ & Calle, pensiones & Cocaína, VI & $\begin{array}{c}\text { VHB, VHC y } \\
\text { VIH }\end{array}$ & CAS y PMM \\
\hline $\begin{array}{l}\text { Esmeralda } \\
\text { Parroquiana }\end{array}$ & Mujer & 1974, Granada & $\begin{array}{l}\text { PNC, hurtos y } \\
\text { trapicheos }\end{array}$ & Piso compartido & $\begin{array}{c}\text { Heroína y } \\
\text { cocaína, VP y } \\
\text { VI }\end{array}$ & $\begin{array}{c}\text { VHB, VHC y } \\
\text { VIH }\end{array}$ & CAS y PMM \\
\hline $\begin{array}{l}\text { Vanesa } \\
\text { Fiestera }\end{array}$ & Mujer & 1973, Barcelona & PNC & $\begin{array}{l}\text { Vivienda } \\
\text { familiar }\end{array}$ & Heroína, VI & $\begin{array}{c}\text { VHB, VHC y } \\
\text { VIH }\end{array}$ & CAS y PMM \\
\hline $\begin{array}{l}\text { Edgar } \\
\text { Rutero }\end{array}$ & Transex & 1974, Barcelona & PNC & Piso compartido & Cocaína, VI & VHC y VIH & CAS y centro de día \\
\hline $\begin{array}{l}\text { Alexis } \\
\text { Parroquian@ }\end{array}$ & Intersex & 1981, Barcelona & $\begin{array}{l}\text { Hurtos y } \\
\text { trapicheos }\end{array}$ & $\begin{array}{l}\text { Vivienda } \\
\text { familiar }\end{array}$ & $\begin{array}{l}\text { Heroína y } \\
\text { cocaína, VI }\end{array}$ & VHC y VIH & CAS y PMM \\
\hline $\begin{array}{l}\text { Dave } \\
\text { Fiestero }\end{array}$ & Varón & 1978, Barcelona & $\begin{array}{c}\text { PNC y } \\
\text { mendicidad }\end{array}$ & Piso compartido & Heroína, VI & VHC & CAS y PMM \\
\hline $\begin{array}{l}\text { Miguel } \\
\text { Fiestero }\end{array}$ & Varón & 1981, Barcelona & PNC y hurtos & $\begin{array}{l}\text { Vivienda } \\
\text { familiar }\end{array}$ & Cocaína, VI & VHC & CAS y PMM \\
\hline $\begin{array}{l}\text { Erika } \\
\text { Pasajera }\end{array}$ & Mujer & $\begin{array}{l}\text { 1983, } \\
\text { Vic }\end{array}$ & Trabajo sexual & $\begin{array}{l}\text { Vivienda } \\
\text { familiar }\end{array}$ & Cocaína, VI & VIH & No \\
\hline $\begin{array}{l}\text { Mick } \\
\text { Fiestero }\end{array}$ & Varón & 1981, Barcelona & Sueldo & $\begin{array}{l}\text { Vivienda } \\
\text { familiar }\end{array}$ & Heroína, VI & NO & CAS y PMM \\
\hline $\begin{array}{l}\text { Jonatan } \\
\text { Rutero }\end{array}$ & Varón & 1984, Barcelona & $\begin{array}{l}\text { PNC, hurtos y } \\
\text { trapicheos }\end{array}$ & Calle & $\begin{array}{l}\text { Heroína y } \\
\text { cocaína, VI }\end{array}$ & VHC y VIH & $\begin{array}{l}\text { Irregular en CAS y } \\
\text { PMM }\end{array}$ \\
\hline $\begin{array}{l}\text { Javier } \\
\text { Rutero }\end{array}$ & Varón & 1985, Barcelona & Mendicidad & Calle & Cocaína, VI & NO & $\begin{array}{c}\text { Irregular en CAS y } \\
\text { PMM }\end{array}$ \\
\hline $\begin{array}{l}\text { Cris } \\
\text { Rutera }\end{array}$ & Mujer & 1985, Barcelona & Mendicidad & Calle & Cocaína, Vl & NO & $\begin{array}{l}\text { Irregular en CAS y } \\
\text { PMM }\end{array}$ \\
\hline $\begin{array}{l}\text { Kike } \\
\text { Parroquiano }\end{array}$ & Varón & $\begin{array}{l}\text { 1978, } \\
\text { Vigo }\end{array}$ & $\begin{array}{l}\text { Ayuda familiar y } \\
\text { trapicheos }\end{array}$ & Piso compartido & Heroína, VP & NO & CAS y buprenorfina \\
\hline $\begin{array}{l}\text { Berta } \\
\text { Parroquiana }\end{array}$ & Mujer & $\begin{array}{c}\text { 1967, } \\
\text { Mollerussa }\end{array}$ & Mendicidad & Calle, pensiones & $\begin{array}{l}\text { Heroína y } \\
\text { cocaína, VI }\end{array}$ & VHC y VIH & CAS y CSMA \\
\hline
\end{tabular}

Nota: CAS = centro de atención y seguimiento a las drogodependencias; CSMA = centro de salud mental de adultos; PMM = programa de mantenimiento con metadona; PNC = paga no contributiva; VHB = virus de la hepatitis B; VHC = virus de la hepatitis C; VIH = Virus de la Inmunodeficiencia Humana; VI = vía intravenosa; $\mathrm{VP}=$ vía pulmonar

\section{Instrumentos y procedimiento}

Se recogieron datos mediante la observación participante y entrevistas semiestructuradas. La observación participante implica observar y participar estableciendo una relación de compromiso/separación que se construye siguiendo las lógicas del trabajo de campo (Hammersley y Atkinson, 1994). Para ello, se interaccionó con los usuarios de las $\mathrm{SCH}$ en las diferentes áreas de los dispositivos. Por ejemplo, se estableció conversación con los usuarios en el momento de realizar el consumo en las SCH, se tomó contacto con ellos en las zonas de reposo y alimentación para proponer la realización de entrevistas o se observaron las interacciones de los profesionales con los usuarios en las consultas de enfermería o en la zona del programa de intercambio de jeringuillas. Además, se realizaron observaciones en los alrededores de las $\mathrm{SCH}$. Para ello se acompañó a los educadores de calle que acuden a las zonas abiertas de consumo para la recogida de jeringuillas sucias o para la captación de usuarios para que acudan a la $\mathrm{SCH}$. Durante estas observaciones se tuvo un mayor conocimiento de las prácticas de consumo y los discursos de los usuarios en sus "espacios 
naturales". Por ejemplo, se observaron las prácticas del consumo en la vía pública (portales, descampados, etc.) y se estableció conversación con los usuarios para conocer los motivos de consumo en estos lugares. El registro de estas sesiones se plasmó en un diario de campo para describir los hechos, los acontecimientos, los lugares y personas, e interpretar las impresiones y las reflexiones del investigador (Emerson, Fretz y Shaw, 2011). En total se realizaron 259 sesiones, redactadas en 1.928 páginas del diario de campo.

Las entrevistas semiestructuradas (DiCicco-Bloom y Crabtree, 2006; Jamshed, 2014) permitieron recoger datos de forma abierta sobre las características de los usuarios. Para ello se utilizó un guión de temas que incluía preguntas deductivas para el análisis de los estilos de vida: a) condiciones socioeconómicas, laborales y jurídico-penales, por ejemplo: “¿De dónde sueles recibir tus ingresos económicos?”, “¿Qué relación mantienes con tu familia?"; b) percepción del estado de salud y seguimiento sanitario, por ejemplo: “¿Qué opinas del tratamiento farmacológico de drogodependencias que sigues actualmente?", “¿Por qué razones tienes dificultades para realizar seguimiento en un centro de salud?"; c) intensidad y formas de consumo de drogas, por ejemplo: "Háblame de tu consumo de drogas actual, ¿Qué drogas consumes y cómo las consumes?", “¿Cuáles son las razones que te llevan a consumir en la calle o en una casa?"; d) uso y actitudes hacia los programas sociosanitarios de los centros con SCH, por ejemplo: “¿Por qué razones acudes a consumir a las $\mathrm{SCH}$ ?" "¿Qué opinión tienes de los profesionales de las SCH?", “¿Qué cambios has observado en tus prácticas de consumo al acudir a la $\mathrm{SCH}$ ?"; e) expectativas de futuro, por ejemplo: "Teniendo en cuenta tu situación actual ¿Qué piensas hacer en un futuro?”, “¿Qué tipo de ayuda crees que necesitarías para mejorar tu situación actual?". Las entrevistas fueron grabadas digitalmente y transcritas textualmente para su posterior análisis. Todos los entrevistados firmaron un consentimiento informado y un documento donde se informaba del objetivo de la investigación y del uso de los datos recogidos. Estos documentos fueron aprobados por la Comisión de ética en la experimentación animal y humana (CEEAH) de la Universitat Autònoma de Barcelona.

\section{Análisis de datos}

Se realizó un análisis de contenido de los materiales recogidos. Este consistió en la interpretación y la comparación objetiva y sistemática de los datos en términos de algún denominador común (Bengtsson, 2016). En primer lugar, se analizaron los datos obtenidos mediante la observación participante y las entrevistas semiestructuradas para construir provisionalmente la tipología de los estilos de vida de los usuarios de las $\mathrm{SCH}$. Seguidamente, para definir cada una de estas categorías de comportamientos se compararon en profundidad las características de los diferentes "tipos sociales" siguiendo unos criterios cualitativos. Para ello, se formalizando unos códigos que permitieron identificar descripciones e ideas que se agruparon en familias temáticas generando categorías, elementos de clasificación de los temas abordados en la investigación. La credibilidad y validez de los datos analizados fueron incrementadas mediante la triangulación (Carter et al., 2014), utilizando diferentes técnicas de obtención de datos (observación participante y entrevistas semiestructuradas) y recogiendo datos en diversos lugares (unidades de observación) y a diferentes usuarios de las $\mathrm{SCH}$.

\section{RESULTADOS}

Los resultados de esta investigación permitieron identificar cuatro estilos de vida de los usuarios de las SCH. La tipología ha sido diseñada mediante la comparación de las cinco dimensiones propuestas por Dumont y Clua (2015). Si bien, en la clasificación de los diferentes estilos de vida se toma como eje central la intensidad - de mayor a menor - de las formas de consumo y la adherencia de los usuarios a los programas de reducción de daños desarrollados en las SCH. Los estilos han sido categorizados como "rutero", "parroquiano", "pasajero" y "fiestero".

\section{"Rutero": usuario en movimiento diario de una sala de consumo a otra}

El "rutero" es un usuario desempleado que ocupa todo el día en ir de un lugar a otro para "buscarse la vida" (mendicidad, pequeños trapicheos, robos y hurtos) y consumir drogas. Es un usuario con pocas redes sociales y familiares que suele vivir en la calle o de forma irregular en pensiones o pisos compartidos. Presenta un aspecto descuidado que le hace ser reconocible por la policía siendo fácilmente identificado y detenido en el caso de infringir la ley. 
El "rutero" es un consumidor con problemas de salud ( $\mathrm{VIH}$, hepatitis B y $\mathrm{C}$, salud mental, etc.) que sus condiciones de vida no le permiten integrar los programas y los tratamientos para un seguimiento favorable de las patologías derivadas del consumo o lo realiza con un bajo cumplimiento. Este tipo de usuario organiza su tiempo entre las diversas zonas de venta de drogas, consumiendo en espacios públicos, pisos de venta de drogas y en las diferentes $\mathrm{SCH}$. Es consumidor de cocaína o speedball (mezcla de heroína y cocaína) por vía parenteral de gran intensidad, que realiza un mínimo de 5 ó 6 consumos diarios. Su preocupación principal es la de obtener dinero por cualquier medio para consumir drogas y evitar el malestar del síndrome de abstinencia.

"Yo me paso el día dando vueltas, los toxicómanos estamos liados las 24 horas. Me busco la vida como puedo. Igual me hago (robo) una tienda o pido dinero en el metro. Tengo alguna causa abierta y no sé cuándo me va tocar entrar en prisión" (Jonatan).

"Llevo tiempo viviendo en la calle, ahora duermo en un cajero con un colega. [...] No siempre consumo en la sala $(\mathrm{SCH})$, depende como me pille o de que esté abierta... a lo mejor está abierta pero prefiero ir a las vías a meterme un chute (inyección) más tranquilo" (Antonio, diario de campo).

Acude a la SCH para consumir, obtener/desechar material de consumo o reposar un breve espacio de tiempo tras el mismo. La relación con los profesionales es instrumental (pedir material de consumo, solicitar el turno de duchas, etc.) y con el resto de usuarios mantiene una relación con fines de crear alianzas para conseguir dinero o para comprar drogas a medias. Dada la intensidad de consumo y las condiciones de vida, es un consumidor que no siempre utiliza los recursos para prevenir las enfermedades infectocontagiosas, como los programas de intercambio de jeringuillas (PIJ), o evitar las sobredosis, como los talleres para la administración de naloxona, manteniendo prácticas de riesgo fuera del contexto de las $\mathrm{SCH}$. Las perspectivas de futuro de este tipo de usuario son las de mejorar su estado físico, manteniendo un control del consumo de drogas que mejore su calidad de vida.

"Mi situación es bastante delicada, quiero dejar de consumir. Sigo consumiendo, sin medicación (antirretrovirales) y con el hígado tocado. Tengo que tomar una decisión, a lo mejor no duro 10 años pero, dentro de lo que dure, que sea lo mejor posible dentro de la condición que la salud me deje" (Edgar).

"Paso el día liándola para conseguir dinero para droga... estoy hecho polvo. Hace mucho que tengo hepatitis C. El bicho ( $\mathrm{VIH})$ no lo tengo. Me estoy haciendo pruebas porque no paro de meterme con uno y con el otro. Ahora no tomo metadona aunque la doctora quiere ponérmela. Tomo medicación para las paranoias, oía voces, me peleaba con la gente. Tengo que hacer algo ya" (Ramón).

De este modo, el "rutero" mantiene un consumo intenso y realiza un uso limitado de los programas de reducción de daños. Este realizará tratamientos y seguimientos de las drogodependencias de forma más rigurosa, así como pedir ayuda para cubrir sus necesidades sociales, en el momento que contemple que el consumo le reporta una serie de déficits físicos y psíquicos y problemas con la ley que le llevarán a reflexionar en acciones para cambiar su estilo de vida.

\section{"Parroquiano": usuario habitual de una sala de consumo}

El "parroquiano" es un usuario que obtiene dinero para comprar drogas a través de rentas mínimas de inserción social pero días concretos del mes los emplea "buscándose la vida" en la ciudad por diferentes métodos (mendicidad, hurtos y pequeños trapicheos). Es un usuario con pocas redes sociales y con cierta desvinculación familiar que tras pasar gran parte del día en una $\mathrm{SCH}$, vuelve a su casa o a refugio urbano tras el cierre de este dispositivo.

Es un usuario que refleja un deterioro físico a consecuencia del consumo desarrollado en un largo periodo de tiempo. Sin embargo, es menos visible que los usuarios "ruteros", manteniendo un aspecto más cuidado y ciertas pautas de higiene. Sigue con regularidad los tratamientos y seguimientos a las drogodependencias prescritos en los centros donde consume o en su centro de atención y seguimiento a las drogodependencias (CAS) de referencia. Suele estar en tratamiento con metadona y consume heroína o cocaína, obtenidas a través de otros usuarios o en puntos de venta cercanos a las $\mathrm{SCH}$, por vía parenteral o pulmonar, en baja intensidad; realiza dos o tres consumos diarios aunque no todos los días. 
"Me meto heroína pero no como antes que andaba todo liado con la coca (cocaína). En estos momentos he conocido gente de aquí del centro (SCH) que me ayuda y ya no ando tan desfasado. Vengo y me ducho, hablo con la trabajadora social, voy a las visitas con la médica... Por el momento, vivo en la calle" (Juanjo).

"Estoy tomando metadona y hago seguimientos con el médico. Me voy haciendo analíticas para ver como tengo la carga viral. También tomo trankimazín y cocaína. Estoy pendiente de hacer un ingreso porque me piden una condena por vender pastillas y algún robo en tiendas. Tengo una entrevista para ir con la trabajadora social y el abogado para una medida alternativa" (Julián).

El "parroquiano" siempre consume en las $\mathrm{SCH}$ cuando están abiertas y mantiene una relación cordial con los profesionales y el resto de usuarios. Este tipo de usuario está altamente comprometido con la actividad del centro: acude a las visitas sanitarias o de tipo social y participa en los talleres de promoción de la salud o en las actividades lúdicas. Su situación empeora cuando cambia la vía de consumo, de la vía pulmonar a la parenteral, abandona la metadona e intensifica el consumo de heroína o sustituye la heroína completamente por metadona y centra su consumo en la cocaína de forma intensa. En su situación actual, toma conciencia de dar un salto cualitativo para mejorar su salud, solicitando tratamientos de mayor exigencia.

"Actualmente, mi idea es dejar la heroína porque nunca estuve en esta movida y esta movida no va conmigo. De aquí a unos años lo veo cada vez más cronificado y cada vez me lo estoy tomando más en serio el tema de dejarlo" (Kike).

"Hace un mes que me diagnosticaron VIH y VHC por compartir jeringuillas. He estado ingresada un mes en el hospital psiquiátrico, por psicosis... de tomar cocaína. Quiero seguir terapia de reinserción social para vincularme a una nueva forma de vivir sin tener que estar constantemente drogada. Marcarme una meta de funcionamiento normal diario" (Berta).
El usuario "parroquiano" refiere el deseo de adoptar un estilo de vida libre de drogas o mantener el actual, evitando malestares relacionados con el consumo de drogas (síndromes de abstinencia, sobredosis e infecciones). Mientras tanto, integra los programas de reducción de daños para no empeorar su estado de salud y se plantea en un futuro realizar con éxito tratamientos para las drogodependencias y los problemas de salud concomitantes con el objetivo de aumentar su calidad de vida, así como llevar una vida normalizada.

\section{"Pasajero": usuario de paso por una sala de consumo}

El "pasajero" es un usuario que dedica la mayor parte del día a la familia, al trabajo y a realizar actividades lúdicas. Obtiene el dinero para consumir drogas de su salario laboral o pequeños trapicheos. Procura ocultar su adicción en el ámbito familiar o laboral. Para ello, evita pasar mucho tiempo en los lugares de venta y consumo de drogas.

Es un usuario que mantiene un aspecto físico y estético desapercibido que le hace ser difícilmente identificado como consumidor de drogas por otros usuarios, policía y transeúntes. Suele acudir siempre a la misma $\mathrm{SCH}$, situada en el trayecto de casa al trabajo o viceversa $u$ otras actividades no relacionadas con el consumo de drogas. Consume heroína, obtenidas en los puntos de venta habituales, preferentemente, por vía pulmonar o nasal en baja cantidad, dos o tres veces al día utilizando siempre material higiénico. No realiza tratamientos para las drogodependencias.

"Yo vengo poco por aquí (SCH). Sólo vengo al salir del curro (trabajo). Estoy en una recepción de un hotel... hablo varios idiomas. Vengo aquí a fumar (heroína por vía pulmonar) porque mi mujer no sabe nada. Piensa que lo he dejado. En casa no puedo hacerlo... no me voy a meter en el baño a meterme una raya" (Rachid, diario de campo).

"He venido un momento aquí ( $\mathrm{SCH}$ ) por he llamado a un colega que me va a traer algo (dosis de droga). Me estoy un rato por aquí y adiós, tengo cosas que hacer" (Manolo, diario de campo).

El "pasajero" es un usuario oculto que procura no visibilizar sus prácticas de consumo estigmatizantes y a continuar con una vida normalizada. Acude a las $\mathrm{SCH}$ sólo para consumir y obtener material para el consumo. 
La relación con los profesionales y el resto de usuarios es discreta y no utiliza apenas el resto de espacios de los centros con $\mathrm{SCH}$. Es un usuario que reflexiona sobre su consumo y las opciones para controlarlo.

"Estoy pensando en dejar Barcelona y volver a Madrid. Llevo un tiempo que estoy tonteando demasiado. Voy a pedir el traslado en mi empresa y a pedir tratamiento para dejar el caballo (heroína). Aquí no estoy haciendo nada. A ver si me quito (desintoxico) y me voy a disfrutar de mi hija que está en Madrid" (Jacinto, diario de campo).

"Siempre he trabajado de una cosa u otra pero consumo cocaína hace años, a veces por vena... el caballo (heroína) casi no lo he tocado. Estuve un tiempo mal pero ahora estoy mejor, vivo con mis abuelos. Ahora llevo unos días que se me ha ido la olla. Espero irme a casa para estar con mis abuelos" (Erika).

El "pasajero" es un usuario que procura no empeorar su situación, intensificando el consumo o realizando consumos por vía parenteral. Por ello, se plantea buscar métodos para controlar el consumo o dejar de consumir. Mientras, se lamenta de su rutina diaria y hace esfuerzos por mantener oculta su adicción, utilizando métodos de prevención y promoción de la salud que no intercedan en su cotidianidad laboral y familiar. Sin embargo, presenta dificultades para compaginar el seguimiento de las drogodependencias con su actividad habitual.

\section{"Fiestero": usuario ocasional en días indicados}

El "fiestero" es un usuario en proceso de recuperación que obtiene ingresos a través de rentas mínimas de inserción social o del sueldo. Vive con la familia y procura ocupar el tiempo libre trabajando o realizando cualquier actividad desvinculada del consumo de drogas.

Se trata de un usuario con problemas de salud crónicos (enfermedades infectocontagiosas, trastornos mentales, etc.) que mantiene un aspecto cuidado, pero con ciertos signos de deterioro debido a un consumo de drogas intenso en el pasado (cicatrices, callos, etc.) que oculta con diferentes estrategias. Realiza tratamientos o terapias para las drogodependencias con rigurosidad en los centros de atención a las drogodependencias u otros dispositivos para el seguimiento de patologías concomitantes. Si bien, acude ocasionalmente a las $\mathrm{SCH}$ para consumir heroína o cocaína por vía parenteral en condiciones higiénicas.

"Yo solo vengo aquí ( $\mathrm{SCH}$ ) los días que cobro el paro... Ilevo unos meses sin trabajar. De momento, vivo con mi madre y miro de no liársela. Voy a mis visitas del médico... si me dieran un currito (trabajo) dejaré de hacer tonterías" (Alberto, diario de campo).

"Vivo con mi madre, mi hermano y mi tía. He intentado trabajar y lo he hecho en varios sitios. He trabajado repartiendo paquetes, en la cadena de producción... Duro poco, tres meses como mucho. Ahora hace tiempo que no trabajo. Tengo una paga por invalidez... hago mis seguimientos médicos $y$ tal. Sólo vengo por aquí de tanto en tanto. Cuando cobro me hago una fiestecilla y ya está" (Miguel).

El "fiestero" es un usuario que utiliza los programas de promoción de la salud de las $\mathrm{SCH}$ para evitar daños derivados del consumo. Si bien, no participa en las diferentes actividades del centro aunque tras el consumo, aprovecha para conversar con otros usuarios o los profesionales conocidos de etapas anteriores cuando el consumo era más intenso y acudía diariamente a la $\mathrm{SCH}$. Es un usuario consciente de su situación que reflexiona en cómo mantener o mejorar su estado físico y psicológico.

"Actualmente, mi apoyo es mi madre por todo.... vino a recogerme cuando estaba tirada en la calle. Mi relación con mi familia está bien. [...] Tomo rivotril para dormir y estoy a 400 defensas (CD4), me han subido. Tomo 5 pastillas, 2 por la mañana y 3 por la noche, Kaletra y Kivexa (antirretrovirales del VIH). Luego, cada jueves voy a terapia pero yo lo que quiero es hacer cursos, me apetece hacer inglés que tengo que ir a mirarlo. Hay que tener la mente ocupada que si no me fumo un porro o algo más y para qué" (Vanesa).

"Fui al médico y le dije que quería bajar la metadona, estaba consumiendo y me dijo de subir la metadona de 60 mgr. a 70 porque estaba consumiendo. Lo que pienso es que la estoy cagando. Para mí las indicaciones están bien, no las discuto para nada. Ahora estoy consumiendo cada 
tres días más o menos desde hace dos meses. He estado tres meses sin nada y hace dos meses caí otra vez. Ahora estoy con la intención de dejarlo otra vez" (Mick).

Los proyectos de futuro de este tipo de usuario es mantener una buena relación familiar y continuar el proceso de recuperación acudiendo a las visitas de los centros de atención a las drogodependencias siguiendo las pautas marcadas por los profesionales. Su mayor preocupación es no recaer en un consumo intensivo, manteniendo consumos ocasionales que no le impida el autocuidado y a continuar con una situación familiar u ocupacional favorable.

\section{DISCUSIÓN Y CONCLUSIONES}

La investigación ha dado como resultado el diseño de cuatro estilos de vida, en los que se ha analizado y comparado las características cualitativas de los diferentes tipos sociales para comprender las diferencias de intensidad del consumo de drogas y de adherencia a los programas de reducción de daños entre los usuarios de drogas que acuden a las SCH de Barcelona.

La elaboración de la tipología, mediante el análisis de cinco dimensiones propuestas por Dumont y Clua (2015), nos indica que los estilos de vida de los usuarios de las $\mathrm{SCH}$ no son definidos como patrones estáticos, sino que éstos pueden cambiar en el tiempo debido a su carácter dinámico. En cada uno de ellos, la transferibilidad y la reversibilidad son posibles por la influencia de las disposiciones y las condiciones de vida que evolucionan a lo largo del tiempo, reflejadas en los cambios de intensidad del consumo y la adherencia a los programas de reducción de daños. Este aspecto nos advierte que la intensidad del consumo no es un efecto exclusivo del potencial adictivo de las sustancias sino a un conjunto de factores socioculturales que contribuyen a tales situaciones (Apud y Romaní, 2016; Bourgois y Schonberg, 2009).

En términos generales, los usuarios de las $\mathrm{SCH}$ consumen drogas para superar los malestares generados en los procesos de socialización y los propios del consumo intensivo que conducen a emprender diferentes itinerarios terapéuticos utilizando los servicios sociosanitarios de los que disponen u otras medidas preventivas. Desde 2001 los usuarios acuden a las SCH como parte del proceso de drogodependencias con el objetivo de mejorar su calidad de vida, controlando o dejando de consumir a diario o para siempre. La toma de decisiones dependerá de la capacidad de agencia y los condicionantes estructurales que definen los estilos de vida. En cada uno de éstos, se detecta que en el momento que las prácticas diarias supongan un daño al colectivo, estas serán cuestionadas y se tomarán posiciones frente a las prácticas con las que se "corren riesgos" (Douglas, 1996). Estas prácticas se pondrán en tela de juicio, llegando a tomar la decisión de cambiar elementos de los estilos de vida. Los usuarios de tipo "rutero", refieren el deseo de buscar diferentes métodos para controlar o dejar el consumo diario, el cual, les reporta una serie de problemas sociosanitarios insostenibles. Los "parroquianos" expresan no querer empeorar su situación y no intensificar el consumo a diario para poder replantearse, en un tiempo, un tratamiento con garantías que aumente su calidad de vida. Los "pasajeros" procuran continuar ocultando su situación actual y tomar medidas para no intensificar el consumo de drogas o, incluso, para alejarse del consumo a diario. Los "fiesteros" refieren tomar medidas para no transformar el consumo ocasional en habitual para continuar cuidándose y no empeorar su situación actual.

La investigación demuestra que las lógicas y prácticas de los usuarios de las $\mathrm{SCH}$ no son carentes de racionalidad sino que son estructuradas para adaptarse según los condicionantes de su cotidianidad. Dicha reflexión debe conducir a no responsabilizar y culpabilizar exclusivamente a los usuarios de las $\mathrm{SCH}$ y a tener un mayor conocimiento del contexto y de las circunstancias inherentes de cada estilo de vida. Son estos últimos los que influyen en la intensidad en el consumo de drogas y en la capacidad de adherencia a los programas preventivos por lo que es fundamental integrar estos aspectos en el análisis para evitar reducir los estilos de vida a una mera descripción de una serie de hábitos o riesgos con efectos en las salud de la población (Menéndez, 1998).

La investigación alerta que el diseño de acciones para la promoción de la salud de los consumidores de drogas debe tener en cuenta la complejidad y la diversidad de los estilos de vida. Desde la salud pública se realizan estudios de carácter estadístico que son insuficientes para dar respuesta a tales condiciones y que 
más bien nos señalan que las diferentes medidas preventivas y de promoción de la salud resultan poco exitosas y/o ineficaces (Suárez, Beltrán y Sánchez, 2006). De este modo, es necesaria la complementariedad con datos cualitativos que otorguen un mayor conocimiento de la racionalidad de la población estudiada y sus contextos éticos, políticos y morales que profundicen en qué estrategias de promoción de la salud pueden llegar a ser más eficaces (Galea, Hall, y Kaplan, 2009; Lopez et al., 2013). Por ello, como apuntan Almeida, Castiel y Ayres (2009) los profesionales, científicos y técnicos no pueden encontrar soluciones unilateralmente siendo necesaria la participación de los sujetos involucrados para reconstruir programas y políticas de manera conjunta.

Los usuarios de las $\mathrm{SCH}$ presentan diferentes características, por lo tanto las intervenciones para mejorar su calidad de vida deben realizarse teniendo en cuenta sus intereses y necesidades inmediatas. En el caso de los "ruteros", usuarios con un consumo intenso con baja adherencia a los programas de reducción de daños, se recomienda ofrecer una mayor flexibilidad en los programas con el objetivo de reducir los riesgos y daños del consumo (sobredosis y transmisión de enfermedades infectocontagiosas) y dar una mayor cobertura de las necesidades sociales básicas. En el caso de los "parroquianos", usuarios con un consumo menos intenso y un fuerte vínculo con la institución, debe darse una mayor respuesta asistencial de las necesidades para aumentar la calidad de vida, así como promover procesos terapéuticos favorables a sus objetivos. En el caso de los "pasajeros", usuarios ocultos que mantienen vínculos sociales, laborales y familiares favorables, se recomienda la promoción de tratamientos flexibles que no intercedan en su actividad diaria para promover una buena adherencia. En el caso de los "fiesteros", usuarios con un consumo ocasional en proceso de recuperación, es necesario llevar a cabo intervenciones específicas que conduzcan a dar un salto cualitativo que evite la cronificación de los procesos de drogodependencias.

El estudio presentado se centró exclusivamente en los estilos de vida de los usuarios de las $\mathrm{SCH}$ de Barcelona. No obstante, los resultados pueden ser transferibles y comparables a otras experiencias y contextos similares. Si bien, la investigación presentó una serie de limitaciones. Una de ellas fue la imposibilidad de realizar observaciones en viviendas o casa okupas, donde se podría haber recogido datos para reflexionar y promover acciones de reducción de daños en estos espacios. Esta limitación se superó parcialmente mediante la realización de entrevistas semiestructuradas en las que se preguntó sobre este aspecto. Otra limitación fue el escaso acceso para entrevistar a usuarios de tipo "pasajero", los cuales acuden a las SCH sólo para consumir en el trayecto de casa al trabajo o viceversa. Más que una limitación, refleja las características específicas de este tipo de usuario que se define por permanecer en un tiempo corto en las $\mathrm{SCH}$; si bien, dicha limitación se ha superado mediante la recolección de datos en el diario de campo. En esta línea, se produjo un acceso limitado a colectivos de consumidores de drogas de países no comunitarios con ciertos problemas de barrera idiomática. Esta limitación denota posibles inconvenientes en la adaptabilidad de los programas en estos colectivos relacionados con las limitaciones lingüísticas que precisa ser estudiada con mayor profundidad como ya se ha apuntado en otro estudio (Sarasa-Renedo et al., 2014). Además, se detectaron fallas en las normas y el funcionamiento de los programas de los centros sociosanitarios con $\mathrm{SCH}$ que no han sido analizadas en profundidad. En esta línea, se sugiere realizar investigaciones que analicen el punto de vista de los usuarios para identificar elementos clave para una mayor adaptabilidad de los programas y promover nuevos enfoques para una mayor cobertura de las necesidades sociosanitarias de los usuarios de las SCH de Barcelona.

En conclusión, esta investigación nos indica la necesidad de implementar estrategias de reducción de daños que se adapten a los estilos de vida de los usuarios de las SCH de Barcelona. En el diseño de éstas, se pone de relieve considerar las diferentes intensidades de consumo de drogas y posibilidades de adherencia a los centros de atención a las drogodependencias, siendo fundamental la flexibilización de los programas y los tratamientos para una mayor cobertura de las necesidades sociosanitarias en la diversidad de usuarios que acuden a estos dispositivos.

\section{REFERENCIAS}

Agència de Salut Pública de Catalunya (ASPCat) (2016). Informe anual 2015. Sistema d'informació sobre drogodependències a Catalunya. Barcelona: Generalitat de Catalunya. 
Almeida, N., Castiel, L.D. y Ayres, J.R. (2009). Riesgo: concepto básico de la epidemiología. Salud Colectiva, 5(3), 323-344.

Álvarez, L.S. (2012). Los estilos de vida en salud: del individuo al contexto. Revista Facultad Nacional de Salud Pública, 30(1), 95-101.

Apud, I. y Romaní, O. (2016). La encrucijada de la adicción. Distintos modelos en el estudio de la drogodependencia. Health and Addictions/ Salud y drogas, 16(2), 115-125.

Bengtsson, M. (2016). How to plan and perform a qualitative study using content analysis. NursingPlus Open, 2, 8-14.

Bourgois, P. y Schonberg, J. (2009). Righteous Dopefiend. Berkeley: University of California Press.

Carlson, R.G., Singer, M., Stephens, R.C. y Sterk, C.E. (2009). Reflections on 40 years of ethnographic drug abuse research: Implications for the future. Journal of Drug Issues, 39(1), 57-70.

Carter, N., Bryant-Lukosius, D., DiCenso, A., Blythe, J. y Neville, A.J. (2014). The use of triangulation in qualitative research. Oncology Nursing Forum, 41(5), 545-547.

Clua, R. (2015). Salas de consumo higiénico en España (2000 - 2013). Salud Colectiva, 11(2), 261-282.

DiCicco-Bloom, B. y Crabtree, BF. (2006). The qualitative research interview. Medical Education, 40(4), 314-321.

Douglas, M. (1996 [1985]). La Aceptabilidad del riesgo según las ciencias sociales. Barcelona: Paidós.

Dumont, G. y Clua, R. (2015). Acercamiento socio-antropológico al concepto de estilo de vida. Aposta Digital. Revista de ciencias sociales, $66,83-99$.

Emerson, R.M., Fretz R. y Shaw, L.L. (2011). Writing ethnographic fieldnotes. Chicago: University of Chicago Press.

Folch, C., Casabona, J., Brugal, M.T., Majó, X., Meroño, M., Espelt, A. y González. V. (2012). Perfil de los usuarios de drogas por vía parenteral que mantienen conductas de riesgo relacionadas con la inyección en Cataluña. Gaceta Sanitaria, 26(1), 37-44.

Galea, S., Hall, C. y Kaplan, G.A. (2009). Social epidemiology and complex system dynamic modelling as applied to health behaviour and drug use research. International Journal of Drug Policy, 20(3), 209-216.

Hammersley, M. y Atkinson, P. (1994 [1983]). Etnografía. Métodos de investigación. Barcelona: Paidós.

Hedrich, D., Kerr, T. y Dubois-Arber, F. (2010). Drug consumption facilities in Europe and beyond. En: Rhodes, T. y Hedrich, D. (Eds.), Harm reduction: evidence, impacts and challenges. Luxemburgo: European Monitoring Centre for Drugs and Drug Addiction, 305-331.

Jamshed, S. (2014). Qualitative research method-interviewing and observation. Journal of Basic and Clinical Pharmacy, 5(4), 87-88.

Lopez, A., Bourgois, P., Wenger, L., Lorvick, J., Martinez, A. y Kral A.H. (2013). Interdisciplinary Mixed Methods Research with Structurally Vulnerable Populations: Case Studies of Injection Drug Users in San Francisco. International Journal of Drug Policy, 24(2),101-109.
Martínez-Salgado, C. (2012). El muestreo en investigación cualitativa: principios básicos y algunas controversias. Ciência y Saúde Coletiva, 17(3), 613-619.

Menéndez, E.L. (1998). Estilos de vida, riesgos y construcción social. Conceptos similares y significados diferentes. Estudios Sociológicos, 16(46), 37-67.

Noël, L., Gagnon, F., Bédard, A. y Dubé, È. (2009). Avis sur la pertinence des services d'injection supervisée: analyse critique de la littérature. Québec: Institut national de santé publique du Québec.

Potier, C., Laprévote, V., Dubois-Arber, F., Cottencin, O. y Rolland, B. (2014). Supervised injection services: What has been demonstrated? A systematic literature review. Drug \& Alcohol Dependence, 145, 48-68.

Rhodes, T. (2009). Risk environments and drug harms: a social science for harm reduction approach. International Journal of Drug Policy, 20(3), 193-201.

Romaní, O. (2010). La epidemiología sociocultural en el campo de las drogas: contextos, sujetos y sustancias. En: Haro J.A. El planteamiento de una epidemiología sociocultural. Un diálogo en torno a su sentido, métodos y alcances. Buenos Aires: Lugar, 89-113.

Sarasa-Renedo, A., Espelt, A., Folch, C., Vecino, C., Majó, X., Castellano, Y., Casabona, J., Brugal, M.T. y Redan Study Group. (2014). Overdose prevention in injecting opioid users: the role of substance abuse treatment and training programs. Gaceta Sanitaria, 28(2), 146-154.

Sepúlveda, M. y Romaní, O. (2013). Conceptualización y políticas de la gestión del riesgo. En: Martínez Oró, D.P. y Pallarés, J. (Eds.), De riesgos y placeres: manual para entender las drogas. Lleida: Milenio, 89-102.

Shaghaghi, A., Bhopal, R.S. y Sheikh, A. (2011). Approaches to Recruiting "Hard-To-Reach" Populations into Research: A Review of the Literature. Health Promotion Perspectives, 1(2), 86-94.

Singer, M. (2012). Anthropology and addiction: An historical review. Addiction, 107(10), 1747-1755

Suárez, R., Beltrán, E.M. y Sánchez, T. (2006). El sentido del riesgo desde la antropología médica: consonancias y disonancias con la salud pública en dos enfermedades transmisibles. Antípoda: Revista de Antropología y Arqueología, 3, 123-154. 